\title{
Geographic Variation in Postoperative Imaging for Low-Risk Breast Cancer
}

\author{
Benjamin L. Franc, MD, MS, MBA; ; Timothy P. Copeland, MPPa; Robert Thombley, BS ${ }^{b}$; Miran Park, PhD; \\ Ben Marafino, BS ; Mitzi L. Dean, MHA, MS ; W. John Boscardin, PhDc; Hope S. Rugo, MDd; \\ David Seidenwurm, MDe; Bhupinder Sharma, FRCR ; Stephen R. Johnston, MA, FRCP, PhD; \\ and R. Adams Dudley, MD, MBA ${ }^{b}$
}

\begin{abstract}
Background: The objective of this study was to examine the presence and magnitude of US geographic variation in use rates of both recommended and high-cost imaging in young patients with early-stage breast cancer during the 18 month period after surgical treatment of their primary tumor. Methods: Using the Truven Health MarketScan Commercial Database, a descriptive analysis was conducted of geographic variation in annual rates of dedicated breast imaging and high-cost body imaging of 36,045 women aged 18 to 64 years treated with surgery for invasive unilateral breast cancer between 2010 and 2012. Multivariate hierarchical analysis examined the relationship between likelihood of imaging and patient characteristics, with metropolitan statistical area (MSA) serving as a random effect. Patient characteristics included age group, BRCA1/2 carrier status, family history of breast cancer, combination of breast surgery type and radiation therapy, drug therapy, and payer type. All MSAs in the United States were included, with areas outside MSAs within a given state aggregated into a single area for analytic purposes. Results: Descriptive analysis of rates of imaging use and intensity within MSA regions revealed wide geographic variation, irrespective of treatment cohort or age group. Increased probability of recommended postoperative dedicated breast imaging was primarily associated with age and treatment including both surgery and radiation therapy, followed by MSA region (odds ratio, 1.42). Increased probability of PET use-a high-cost imaging modality for which postoperative routine use is not recommended in the absence of specific clinical findings-was primarily associated with surgery type followed by MSA region (odds ratio, 1.82). Conclusions: In patients with breast cancer treated for low-risk disease, geography has effects on the rates of posttreatment imaging, suggesting that some patients are not receiving beneficial dedicated breast imaging, and high-cost nonbreast imaging may not be targeted to those groups most likely to benefit.
\end{abstract}

J Natl Compr Canc Netw 2018;16(7):829-837 doi: 10.6004/jncen.2018.7024

Over a number of years or decades, the nearly 3 million US patients with breast cancer who have survived their initial treatment phase will be imaged to screen for additional primary breast cancers and to evaluate for evidence of recurrent or metastatic disease. ${ }^{1}$ Optimizing imaging in these patients is critical to their health and to the welfare

\footnotetext{
aDepartment of Radiology and Biomedical Imaging, ' $P$ Philip R. Lee Institute for Health Policy Studies, Center for Healthcare Value, 'Department of Medicine, Epidemiology \& Biostatistics, and dDepartment of Medicine, University of California, San Francisco, California; 'Diagnostic Imaging, Sutter Medical Group, Sacramento, California; and 'The Royal Marsden NHS Foundation Trust, Chelsea, London, United Kingdom.

Submitted December 15, 2017; accepted for publication March 12, 2018.

Dr. Seidenwurm has disclosed that he has equity in Sutter Medical Group. The remaining authors have disclosed that they have no financial interests, arrangements, affiliations, or commercial interests with the manufacturers of any products discussed in this article or their competitors.
}

of the health system. The American College of Radiology recommends that women with personal histories of breast cancer and dense breast tissue, or breast cancer diagnosed by age 50 years, receive dedicated breast MRI annually. ${ }^{2}$ ASCO and NCCN have issued guidelines stating that, after initial treatment, surveillance for recurrence in pa-

Author contributions: Study design, management, analysis, and/or implementation: Franc, Dean, Rugo, Seidenwurm, Sharma, Johnston, Dudley. Data programming and guidance: Copeland, Thombley, Park, Marafino. Project coordination and agreements: Dean. Statistical analysis: Boscardin. US clinical oncology direction: Rugo. UK clinical oncology direction: Johnston. US radiologic quality and value perspective: Seidenwurm. UK radiologic quality and value perspective: Sharma. Manuscript preparation: Franc, Copeland, Thombley, Park, Marafino, Rugo, Seidenwurm, Sharma, Johnston, Dudley.

Correspondence: Benjamin L. Franc, MD, MS, MBA, Department of Radiology and Biomedical Imaging, Center for Healthcare Value, University of California, San Francisco, 3333 California Street, Suite 265, San Francisco, CA 94118. Email: Benjamin.Franc@ucsf.edu 
Franc et al

tients with stage I-III breast cancer should include periodic physical examination and mammography, but that, in the absence of symptoms, other imaging tests are not beneficial for patients at low risk of recurrence. ${ }^{3-6}$ Although imaging beyond dedicated breast MRI or mammography can be used to detect cancer recurrence before it produces symptoms or signs, ${ }^{7}$ large randomized controlled trials, the first of which were published in the 1990s, ${ }^{8-11}$ and Cochrane database systematic reviews ${ }^{12,13}$ have repeatedly found that such nonbreast surveillance offers no benefit in terms of survival or quality of life for patients with stage I-III (nonmetastatic) disease. ${ }^{3-6}$

The goal of this study was to examine US geographic variation in use rates of postoperative, recommended, dedicated breast imaging and high-cost whole-body imaging with CT, MRI, PET, and/or bone scan-modalities capable of identifying recurrent or metastatic disease in patients with breast cancer receiving treatment consistent with low risk of recurrence or metastases. Identifying geographic regions with low rates of recommended postoperative imaging would allow interventions to target patients not receiving beneficial screening. Identifying geographic regions with high rates of whole-body postoperative imaging, regardless of patient risk of recurrent disease, could focus interventions to reduce unnecessary surveillance imaging.

\section{Methods}

This analysis, approved by the University of California, San Francisco Institutional Review Board, retrospectively assessed geographic variations in the likelihood of receiving postoperative dedicated breast imaging and whole-body surveillance imaging at the metropolitan statistical area (MSA) level. MSAs are geographic regions defined by the Office of Management and Budget and are commonly used by medical claims payers for statistical purposes. A complementary descriptive analysis of variation across broad geographic regions (Northeast, North Central, South, and West) was also performed.

\section{Data Sources}

We used HIPAA-compliant deidentified patientlevel inpatient, outpatient, and outpatient pharmacy claims data from the Truven Health MarketScan Commercial Database, representing the claims of employees and dependents on large employer health benefit programs between January 1, 2010, and December 31, 2013. The claims database originated from approximately 100 insurance companies, including both insurance providers and third-party administrators. The plans represented include a variety of fee-for-service, preferred provider organizations, and capitated health plans. Claims from Medicare, Medicaid, and workers compensation were not included. All new breast cancer diagnoses in the 2010 SEER database were used in a sensitivity analysis of stage at time of diagnosis across regions.

\section{Data Extraction and Cleaning, Calculation of Rates, and Geographic Aggregation}

A total of 38,424 women were identified with claims indicating a breast biopsy between January 1, 2010, and March 30, 2012, followed by primary surgical treatment and 18 additional months of postdiagnosis claims (see supplemental eAppendix 1 for Current Procedural Terminology [CPT] codes, available with this article at JNCCN.org). To limit the study to patients who had treatment compatible with earlystage disease, 2,379 patients receiving neoadjuvant or nonhormonal adjuvant therapy (any chemotherapy or trastuzumab; supplemental eAppendix 2) between initial biopsy and 18 months postsurgery were excluded from further analysis, resulting in a study cohort of 36,045 women.

To evaluate groups of patients who received comparable treatment regimens, treatment cohorts were classified via CPT codes based on the combination of breast surgery type and whether the patient received radiation therapy (RT) (supplemental eAppendix 1). These patient groups were then classified into subgroups based on whether they received postsurgical hormonal therapy (supplemental eAppendix 2), resulting in 12 treatment cohorts.

Claims for body CT, brain CT, PET, body MRI, breast MRI, brain MRI, mammography, and wholebody bone scans in the 18 months after surgery were identified (see supplemental eAppendix 3 for imaging CPT codes). Aside from modalities for dedicated breast imaging (ie, mammography and breast MRI), postoperative imaging claims were only included in the study when the claim included an indication of breast cancer (ICD-9 codes 174.0-174.9).

Patient characteristics examined included age group, whether RT was received, whether hormonal 
therapy was received, payer class, and MSA. Patients residing outside of an MSA were grouped by state into 47 state-based categories and included within the MSA variable, for a total of 428 geographic regions.

\section{Cross Validation of Imaging Rates}

We validated rates of mammographic imaging at the state level by grouping mammographic rates by state and comparing the mean rate of receiving mammography against 2013 rates reported by NCI. ${ }^{14}$ We also compared the mean rates of mammography from our data set for each year studied to those reported by the CDC for the same year. ${ }^{15}$

\section{Data Analysis}

Main Analysis: We conducted a descriptive analysis of imaging use, defined as a patient receiving at least one imaging study, and imaging intensity, defined by mean number of imaging examinations per imaged patient in a 1-year period. Proportions of patients imaged and mean images per imaged patient were compared simultaneously between treatment cohorts and between Northeast, North Central, South, and West US regions. The variations in proportion of patients receiving imaging between regions were assessed with chi-square tests, whereas variations in mean annualized number of imaging examinations per imaged patient by region were assessed using Kruskal-Wallis test.

To study mean imaging use and intensities within MSA regions, proportions of patients receiving at least one dedicated breast image or at least one nonbreast tomographic image (ie, any CT, MRI, PET/ CT, or bone scan) within each MSA region were determined by age group and treatment cohort. Mean nonbreast tomographic imaging intensity within MSA regions was also determined by age group and treatment cohort.

To estimate independent predictors of imaging use, we created 2-level hierarchical models with MSA as a random effect to account for clustering of patients by geographic region. Age group, RT, surgical therapy, drug therapy, and payer type were included as fixed effects. Binary outcome variables assessed with this model include use of brain CT, brain MRI, body CT, body MRI, body PET/CT, bone scan, breast MRI, mammography, tomographic nondedi- cated breast imaging, and tomographic brain imaging (ie, any brain CT or MRI).

The influence of MSA was summarized by the median odds ratio (OR) - the median ratio of the odds of imaging use between equivalent patients with breast cancer of 2 randomly selected MSAs, with the clusters compared in descending order so ORs consistently exceed 1. ${ }^{16,17}$ Median OR was computed from the random effects' estimated variance and was directly comparable to fixed-effect variables' ORs. A fixed effect-only model was compared with the multilevel hierarchical model, and C-statistics were calculated for each model. Models were not reported if C-statistics did not yield acceptable discrimination (ie, a C-statistic of at least 0.7)..$^{18}$

Analysis was performed using SAS 9.4 (SAS Institute, Inc.) for sample selection and StataMP 13.1 (StataCorp LP) for statistical analysis.

Geographic Distribution of Stage II-III Breast Cancer: To exclude variation in geographic occurrence of locally aggressive operable cancers as an explanation for any observed geographic variation in imaging rates, the significance of differences in distribution of stage II-IIIA breast cancers diagnosed in women aged 18 to 44 years across regions was estimated. Data from regions defined in the SEER database from 2010 was regrouped to reflect the geographic areas studied in the MarketScan-derived cohort. Chi-square tests assessed variation between the rate of stage IIA/B or IIIA cancers diagnosed across regions.

\section{Results}

\section{Cross-Validation of Imaging Rates}

Cross-validation of state-level mammography rates among patients aged $\geq 40$ years showed that state mammographic rates in our study and 2014 state rates reported by NCI did not differ significantly ( $t$-test $P=.11$; supplemental eTable 1$)$. The analysis of mammographic rate did not include Hawaii, because the number of patients $(n=7)$ enrolled in employer-based plans included in the MarketScan data for Hawaii were insufficient for meaningful analysis. The CDC's 2013 mammography rates were comparable to study rates in women aged 40 to 49 years $(59.6 \%$ vs $64.7 \%$, respectively) and in women aged 50 to 64 years ( $71.4 \%$ vs $73.9 \%$, respectively). ${ }^{19}$ Overall, this validation of our study data seems to 
Franc et al

indicate that our findings are likely to approximate national trends.

\section{Main Findings}

Of 36,045 women aged 18 to 64 years diagnosed with unilateral invasive primary breast cancer, treated with surgery, and not exposed to neoadjuvant/adjuvant chemotherapy or trastuzumab within 18 months of diagnosis, 24,802 (68.8\%) underwent at least one screening or diagnostic mammogram, 4,602 (12.8\%) had at least one breast MRI, 11,418 (31.7\%) had at least one high-cost imaging procedure, and 4,490 $(12.5 \%)$ had at least one PET. A total of 25,501 women $(70.8 \%)$ had at least one dedicated breast image (ie, at least one mammogram or breast MRI). Patient characteristics are described in Table 1.

Table 2 describes the variations in imaging use (ie, use of at least one imaging modality) and intensity (ie, mean annualized number of imaging examinations per imaged patient). Chi-square and Kruskal-Wallis tests revealed significant variation in both use and frequency between regions within each of the treatment cohorts $(P<.05)$. Imaging use and intensity were heterogeneous between cohorts within regions and between regions within treatment cohorts. Notably, among the lowest-risk patients (surgery only), approximately 50\% indicated that they received mammography in the 18 months after initial treatment. Between $64 \%$ and $70 \%$ of patients who received a mastectomy and RT, presumably a group with higher risk, were imaged using a dedicated breast modality. Proportions of patients imaged and mean images per imaged patient also showed heterogeneity at the geographic levels of Northeast, North Central, South, and West regions of the United States (supplemental eTable 2; see eAppendix 4 for states).

The multivariate multilevel hierarchical models of imaging use in the 18 months post-breast surgery revealed 4 modality models with acceptable discrimination: mammography with fixed effects for surgery/ RT combination only, mammography, PET, and dedicated breast imaging (Table 2). The combination of breast surgery type with/without RT was among the strongest predictors of imaging use in all models, to the extent that a model using only a surgery/RT combination as an independent predictor of mammography had a relatively small difference in model discrimination when compared with the mammography model including all independent variables

\begin{tabular}{|c|c|c|}
\hline Patient Characteristics & $\mathbf{N}$ & $\%^{a}$ \\
\hline \multicolumn{3}{|l|}{ Sex } \\
\hline Female & 36,045 & 100 \\
\hline \multicolumn{3}{|l|}{ Surgery + RT combinations } \\
\hline Lumpectomy without RT & 6,196 & 17.2 \\
\hline Lumpectomy + RT & 12,460 & 34.6 \\
\hline Mastectomy without RT & 9,221 & 25.6 \\
\hline Mastectomy + RT & 3,455 & 9.6 \\
\hline $\begin{array}{l}\text { Mastectomy + contralateral prophylactic } \\
\text { mastectomy without RT }\end{array}$ & 3,558 & 9.9 \\
\hline $\begin{array}{l}\text { Mastectomy + contralateral prophylactic } \\
\text { mastectomy + RT }\end{array}$ & 1,155 & 3.2 \\
\hline \multicolumn{3}{|l|}{ Age at surgery, $y$} \\
\hline $18-34$ & 978 & 2.7 \\
\hline $35-44$ & 6,110 & 17.0 \\
\hline $45-54$ & 14,823 & 41.1 \\
\hline $55-64$ & 14,134 & 39.2 \\
\hline \multicolumn{3}{|l|}{$\mathrm{RT}$} \\
\hline Received RT & 18,975 & 52.6 \\
\hline Did not receive RT & 17,070 & 47.4 \\
\hline \multicolumn{3}{|l|}{ Pharmaceutical treatment } \\
\hline Hormonal therapy only & 18,654 & 51.8 \\
\hline No chemotherapy or hormones & 17,391 & 48.3 \\
\hline \multicolumn{3}{|l|}{ Payer class } \\
\hline PPO & 22,253 & 61.7 \\
\hline Comprehensive & 877 & 2.4 \\
\hline EPO & 673 & 1.9 \\
\hline HMO & 3,830 & 10.6 \\
\hline POS & 2,418 & 6.7 \\
\hline POS with capitation & 229 & 0.6 \\
\hline $\mathrm{CDHP}$ & 2,011 & 5.6 \\
\hline HDHP & 1,283 & 3.6 \\
\hline Unknown & 2,471 & 6.9 \\
\hline \multicolumn{3}{|l|}{ Region $^{b}$} \\
\hline Northeast & 7,519 & 20.9 \\
\hline North Central & 8,072 & 22.4 \\
\hline South & 14,139 & 39.2 \\
\hline West & 6,315 & 17.5 \\
\hline
\end{tabular}

Abbreviations: CDHP, consumer-driven health plan; EPO, exclusive provider organization; $\mathrm{HDHP}$, high-deductible health plan; HMO, health maintenance
organization; POS, point of service; PPO, preferred provider organization; RT, radiation therapy.

${ }^{a}$ All percent values are column percentages.

bThe MarketScan database grouped patients into 4 large regions within the United States (Northeast, North Central, South, and West) based on state of residence (see supplemental eAppendix 4).

(C-statistic, 0.85 vs 0.87 ). PET imaging was much more common among women who were treated with single mastectomy with RT (OR, 6.66) and double mastectomy with RT (OR, 6.87) compared with the reference class of lumpectomy without RT.

Each successive age group was more likely to have dedicated breast imaging and less likely to have PET imaging than the reference class of age 18 to 34 years. Compared with the reference class of women with $\mathrm{PPO}$ insurance plans, those with $\mathrm{HMO}$ plans were the least likely to have postoperative dedicated breast imaging of all insurance classes (OR, 0.64). 
Postoperative Imaging Rates in Breast Cancer

\section{Table 2. Characteristics Associated With Postoperative Imaging by Modality}

\begin{tabular}{|c|c|c|c|c|}
\hline & $\begin{array}{l}\text { Mammography (Surgery/RT } \\
\text { Combination Model) }\end{array}$ & Mammography & $\begin{array}{l}\text { Any Dedicated } \\
\text { Breast Imaging }\end{array}$ & PET/CT Imaging \\
\hline Characteristic & OR $(95 \% \mathrm{Cl})$ & OR $(95 \% \mathrm{Cl})$ & OR $(95 \% \mathrm{Cl})$ & OR $(95 \% \mathrm{Cl})$ \\
\hline \multicolumn{5}{|l|}{ Surgery + RT combinations } \\
\hline Lumpectomy without RT & Ref & Ref & Ref & Ref \\
\hline Lumpectomy + RT & $2.61(2.35-2.91)$ & $2.49(2.23-2.78)$ & $2.59(2.30-2.91)$ & $1.75(1.55-1.98)$ \\
\hline Mastectomy without RT & $0.16(0.15-0.18)$ & $0.16(0.15-0.17)$ & $0.16(0.15-0.18)$ & $1.94(1.71-2.20)$ \\
\hline Mastectomy + RT & $0.26(0.24-0.29)$ & $0.25(0.23-0.28)$ & $0.25(0.22-0.28)$ & $6.66(5.84-7.59)$ \\
\hline $\begin{array}{l}\text { Mastectomy + contralateral prophylactic } \\
\text { mastectomy without RT }\end{array}$ & $0.01(0.01-0.02)$ & $0.01(0.01-0.01)$ & $0.02(0.02-0.02)$ & $1.80(1.55-2.10)$ \\
\hline $\begin{array}{l}\text { Mastectomy + contralateral prophylactic } \\
\text { mastectomy + RT }\end{array}$ & $0.02(0.02-0.03)$ & $0.02(0.02-0.03)$ & $0.03(0.02-0.03)$ & $6.87(2.65-3.27)$ \\
\hline \multicolumn{5}{|l|}{ Age at surgery, $y$} \\
\hline $18-34$ & - & Ref & Ref & Ref \\
\hline $35-44$ & - & $1.72(1.44-2.05)$ & $1.62(1.37-1.93)$ & $0.92(0.76-1.12)$ \\
\hline $45-54$ & - & $2.27(1.92-2.69)$ & $2.05(1.74-2.42)$ & $0.86(0.72-1.03)$ \\
\hline $55-64$ & - & $2.66(2.24-3.15)$ & $2.33(1.97-2.75)$ & $0.85(0.71-1.11)$ \\
\hline \multicolumn{5}{|l|}{ Pharmaceutical treatment } \\
\hline No pharmaceutical treatment & - & Ref & Ref & Ref \\
\hline Received hormonal therapy & - & $1.42(1.34-1.5)$ & $1.41(1.33-1.49)$ & $1.03(0.97-1.11)$ \\
\hline \multicolumn{5}{|l|}{ Payer class } \\
\hline PPO & - & Ref & Ref & Ref \\
\hline Comprehensive & - & $0.91(0.74-1.11)$ & $0.92(0.75-1.13)$ & $1.11(0.88-1.38)$ \\
\hline EPO & - & $1.13(0.9-1.41)$ & $1.13(0.9-1.41)$ & $0.91(0.71-1.16)$ \\
\hline HMO & - & $0.63(0.57-0.69)$ & $0.64(0.58-0.71)$ & $0.91(0.80-1.02)$ \\
\hline POS & - & $0.85(0.75-0.96)$ & $0.86(0.76-0.98)$ & $1.03(0.89-1.18)$ \\
\hline POS with capitation & - & $1.11(0.76-1.63)$ & $1.13(0.77-1.66)$ & $1.03(0.68-1.55)$ \\
\hline CDHP & - & $0.87(0.77-0.99)$ & $0.86(0.76-0.98)$ & $1.19(1.03-1.37)$ \\
\hline HDHP & - & $1.04(0.88-1.22)$ & $1.08(0.92-1.28)$ & $1.01(0.84-1.22)$ \\
\hline Unknown & - & $0.79(0.69-0.91)$ & $0.82(0.71-0.94)$ & $1.17(0.99-1.38)$ \\
\hline \multicolumn{5}{|l|}{ Region } \\
\hline Modified MSA (MOR) ${ }^{\mathrm{a}}$ & - & $1.46(1.38-1.54)$ & $1.42(1.35-1.51)$ & $1.82(1.70-1.97)$ \\
\hline C-statistic for area under ROC & 0.85 & 0.87 & 0.87 & 0.73 \\
\hline
\end{tabular}

Abbreviations: CDHP, consumer-driven health plan; EPO, exclusive provider organization; HDHP, high-deductible health plan; HMO, health maintenance organization; MOR, median odds ratio; MSA, metropolitan statistical area; OR, odds ratio; POS, point of service; PPO, preferred provider organization; ROC, receiver operating characteristic; RT, radiation therapy.

${ }^{a}$ The influence of MSA was summarized by the MOR, which is the median ratio of the odds of contralateral prophylactic mastectomy between equivalent patients of 2 randomly selected MSAs $(n=428)$.

Median ORs for MSA regions ranged from 1.42 for any dedicated breast imaging (ie, the odds of postoperative dedicated breast imaging between 2 patients in different MSAs varied by a factor of 1.42) to 1.82 for PET/CT imaging, with similar findings in patients who had received RT and/or hormonal therapy. Figure 1 details the variation in adjusted rates of dedicated breast imaging by MSA region from the dedicated breast imaging model in Table 2 .

In evaluating mean imaging use and intensities within MSA regions, proportions of patients receiving at least one dedicated breast image or at least one nonbreast tomographic image within each MSA region were described with box plots by age group (Figure 2A) and treatment cohort (Figure 2B). Mean nonbreast tomographic imaging intensity within MSA regions was also described with box plots by age group (Figure 3A) and treatment cohort (Figure 3B).
Figure 2A displays wider variation in imaging use in younger age groups, with median proportions of women receiving nonbreast tomographic imaging within MSA regions decreasing slightly with age. Among treatment cohorts (Figure 2B), the variation between MSA regions displayed wide variation across regions for each patient treatment cohort.

The median MSA's mean annualized number of nonbreast tomographic imaging examinations per imaged patient remained approximately 1 in all age groups (Figure 3B), with smaller interquartile ranges in older cohorts. Irrespective of treatment cohort (Figure 3B), the median MSA's mean annualized number of nonbreast tomographic imaging examinations per imaged patient ranged from approximately 0.5 to 1.5 . Mean imaging intensity varied widely, with numerous outliers above the 75 th percentile in each treatment cohort. 


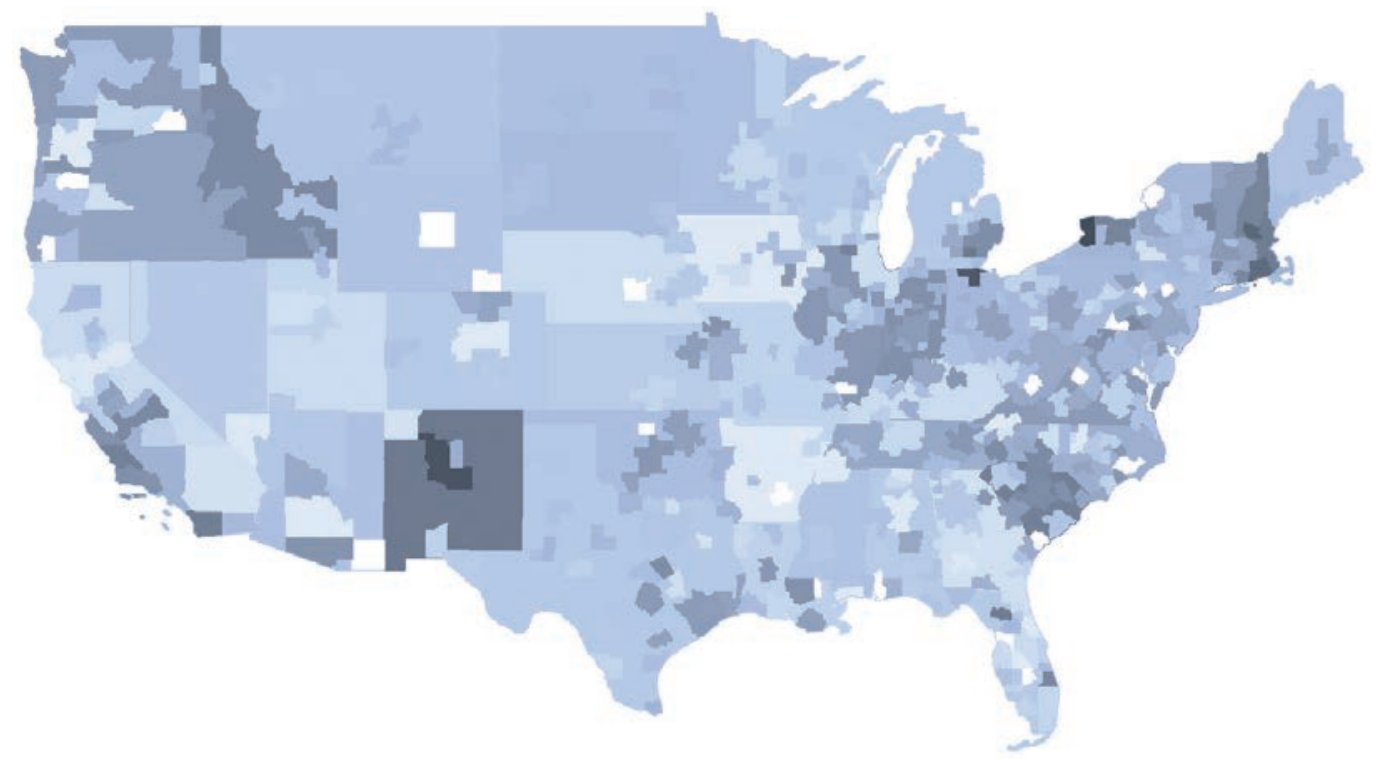

Adjusted probability of dedicated breast imaging after surgery

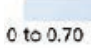
0.75 0.80 0.85 0.90

Figure 1. Adjusted probability of dedicated breast imaging in the 18 months after surgery by metropolitan statistical area.

\section{Underlying Differences in Geographic Distribution of Stage II-III Breast Cancer}

When 2010 SEER data for patients with breast cancer aged 18 to 44 years were grouped into Northeastern, North Central, South, and West regions used in the MarketScan data set, the variation between regions in the rate of stage IIA/B and IIIB breast cancers was not significant $(\mathrm{n}=28,957 ; \mathrm{P}=.371)$.

\section{Discussion}

In this national assessment of postoperative imaging variations in patients with breast cancer who received care compatible with early-stage disease, we found wide geographic variation in imaging use and frequency between MSA regions for both dedicated breast imaging and nonbreast tomographic imaging, presumed to represent surveillance imaging for the detection of recurrent or metastatic disease (Figures 1-3). No MSA-level correlation was observed between rates of breast and nonbreast imaging.

Delivering appropriate screening while avoiding unnecessary postoperative imaging in the millions of US patients with breast cancer has significant clinical, ethical, and economic ramifications. ${ }^{1}$ Oncology imaging costs are increasing at twice the rate of total cancer care, accounting for $4.6 \%$ of the $\$ 32.1$ billion in Medicare cancer expenditures in 2009. ${ }^{20-22}$

Expert guidelines support routine mammography or, depending on age and other factors, dedicated breast MRI for all patients with a personal history of breast cancer. ${ }^{2-6}$ Our study demonstrates a high geographic-based variability regarding whether patients actually receive dedicated breast imaging after treatment for a primary breast cancer.

On the other hand, other nonbreast surveillance imaging to detect recurrent or metastatic disease has consistently shown no benefit in terms of survival or quality of life for patients with stage I-III (nonmetastatic) disease. ${ }^{8-13}$ A recently published meta-analysis of outcomes for patients with early-stage breast cancer showed that recurrences continued at a similar rate through 20 years and that the risk of distant metastasis ranged from $10 \%$ to $41 \%$, correlating with the original $\mathrm{T}$ and $\mathrm{N}$ status. ${ }^{23}$ Pursuing a surveillance strategy apart from dedicated breast imaging early after completion of primary therapy in early-stage cancer with low $\mathrm{T}$ and $\mathrm{N}$ status does not make sense, particularly when the patient may require symptom-driven imaging studies for 20 years after completion of 5 years of tamoxifen. Depending on treatment and geographic location, our study found that $18 \%$ to $46 \%$ of patients received high-cost tomographic imaging within the 


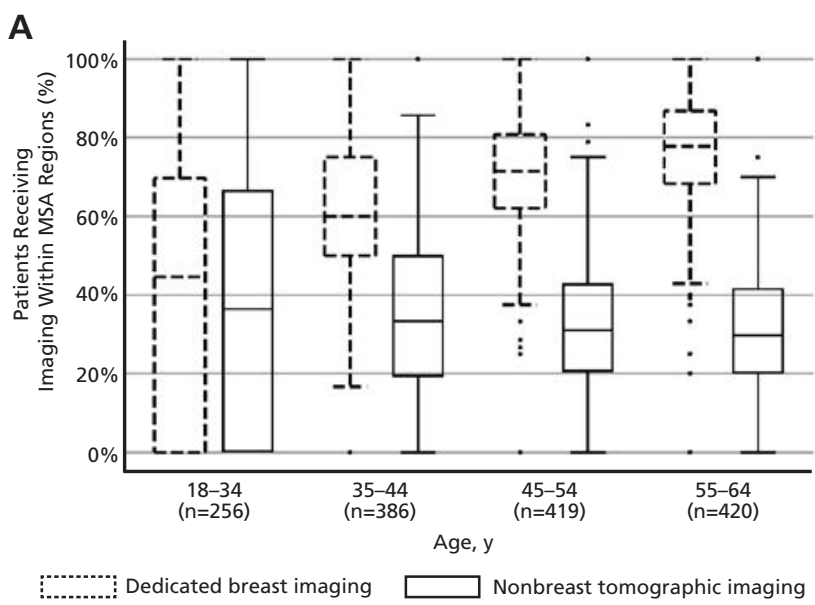

B

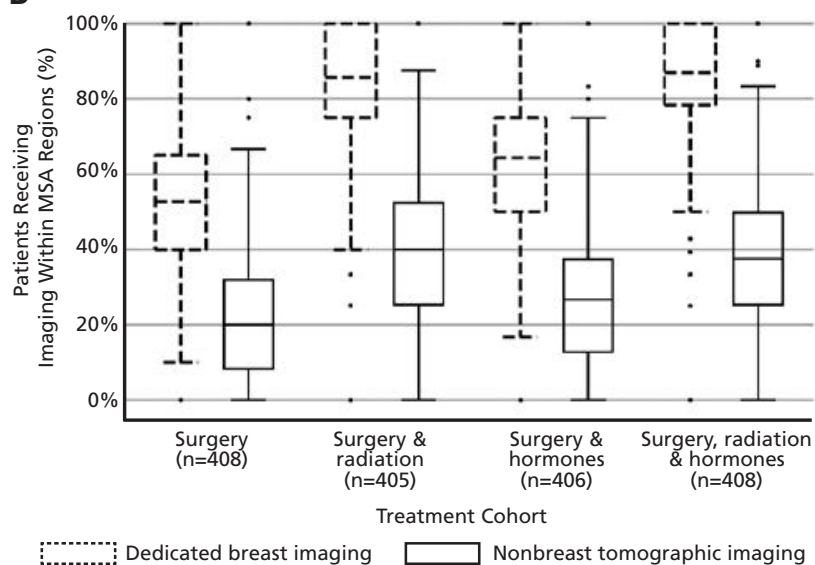

Figure 2. Proportion of patients imaged within metropolitan statistical area (MSA) regions by modality category, grouped by (A) age and (B) treatment cohort.

18 months after surgery. National data on patients with early-stage breast cancer diagnosed in 19982003 showed that $40 \%$ had at least one advanced imaging test (eg, CT or PET) in the 4 years after diagnosis. ${ }^{24}$ Findings from recent smaller studies suggest even higher rates of nonbreast surveillance testing..$^{25,26}$ In some cases, young women may be subject to overly aggressive monitoring strategies because of the presumed risk of age rather than a specific genotype, a practice discouraged by oncology experts. ${ }^{27}$

Surveillance strategies can present medical and financial harms to patients. False-positives can result in unnecessary workup, which can in turn lead to patient anxiety and risk of additional procedures and radiation exposure. ${ }^{28-33}$ As many as $0.4 \%$ of all cancers are estimated to be caused by radiation from imaging, with the time between radiation exposure and clinical detection of a cancer being $\geq 25$ years in some cases. ${ }^{34,35}$
With the aim of improved cancer screening and follow-up, some systems of care have made significant efforts to coordinate care between primary care providers and oncologists, the results of which have yet to be determined. ${ }^{36,37}$ Fear of cancer recurrence in certain patient populations may decrease recommended breast imaging activities or increase highcost whole-body imaging in early-stage breast cancer, both potentially ameliorable through education. ${ }^{38}$

\section{Limitations, Caveats, and Strengths}

Staging information was unavailable, but care was taken to identify only patients with treatment consistent with early-stage disease. Many tumor and patient characteristics contribute to the overall perceived risk of future recurrence or contralateral malignancy, and thereby reflect the type of initial treatment, arguably more so than merely stage alone. ${ }^{39-48}$

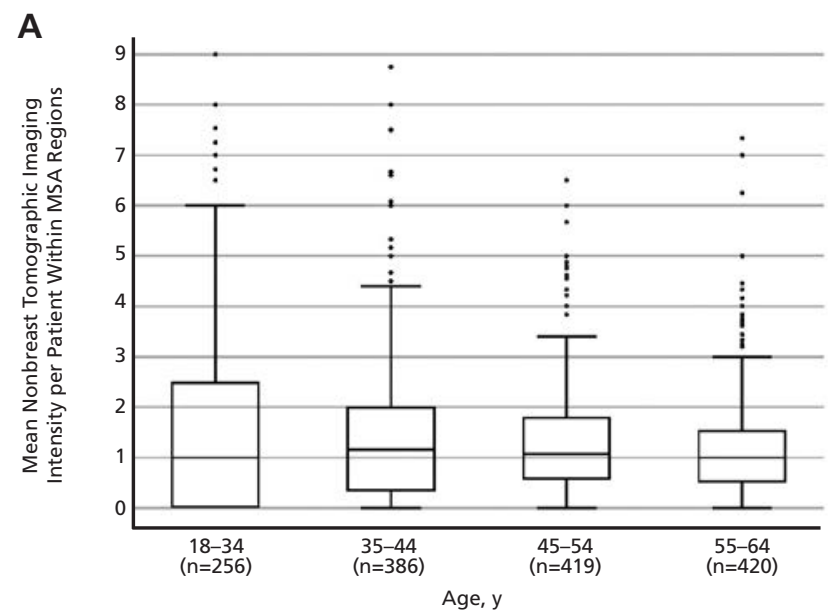

B

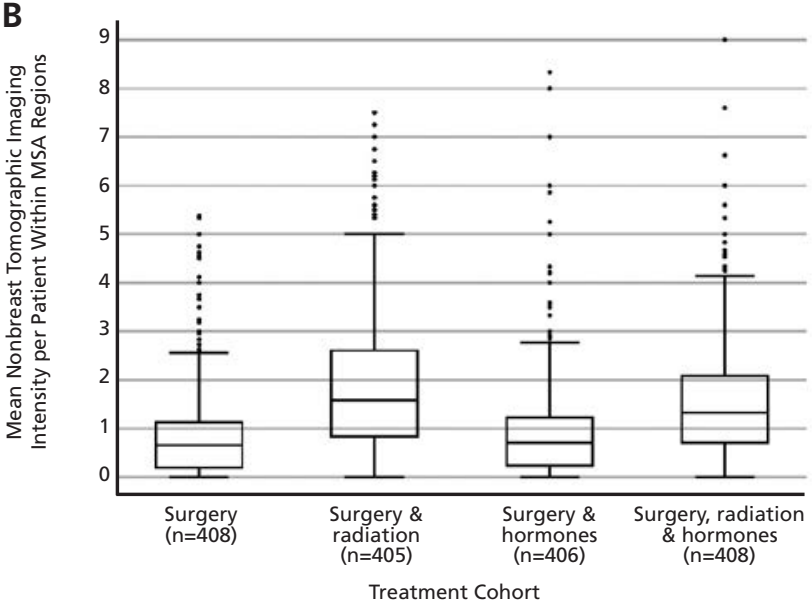

Figure 3. Mean patient nonbreast tomographic imaging intensity (annualized number of imaging examinations per imaged patient) within metropolitan statistical area (MSA) regions, grouped by (A) age and (B) treatment cohort. 
Claims data do not provide information about patients' conditions leading to imaging, and therefore high-cost body imaging in some patients may have been indicated by symptoms and not simply represent whole-body surveillance scanning, or may have been conducted for reasons unrelated to cancer in some cases. We know of no reasons that these conditions would be unevenly distributed geographically, suggesting that our findings are likely relevant despite this limitation.

\section{Conclusions}

The large geographic variation we observed in rates of both postoperative breast imaging and high-cost imaging for detection of recurrent or metastatic disease suggests that some patients are not receiving beneficial screening and that high-cost imaging may not be targeted to the groups most likely to benefit. Some patients are likely receiving excessive amounts of high-cost whole-body imaging, which is not typically indicated in low-risk disease.

\section{References}

1. SEER Stat Facts: Female Breast Cancer. National Cancer Institute/ Surveillance, Epidemiology, and End Results Program website. Available at: http://seer.cancer.gov/statfacts/html/breast.html. Accessed June 10, 2018.

2. Monticciolo DL, Newell MS, Moy L, et al. Breast cancer screening in women at higher-than-average risk: recommendations from the ACR. J Am Coll Radiol 2018;15:408-414.

3. Smith TJ; American Society of Clinical Oncology. The American Society of Clinical Oncology recommended breast cancer surveillance guidelines can be done in a routine office visit. J Clin Oncol 2005;23:6807.

4. Khatcheressian JL, Hurley P, Bantug E, et al. Breast cancer follow-up and management after primary treatment: American Society of Clinical Oncology clinical practice guideline update. J Clin Oncol 2013;31:961965.

5. Gradishar WJ, Anderson BO, Aft R, et al. NCCN Clinical Practice Guidelines in Oncology: Breast Cancer. Version 1.2018. Accessed June 10, 2018. To view the most recent version of these guidelines, visit NCCN.org.

6. Aebi S, Davidson T, Gruber G, et al. Primary breast cancer: ESMO Clinical Practice Guidelines for diagnosis, treatment and follow-up. Ann Oncol 2011;22(Suppl 6):vi12-24.

7. Sharma B, Martin A, Stanway S, et al. Imaging in oncology-over a century of advances. Nat Rev Clin Oncol 2012;9:728-737.

8. Palli D, Russo A, Saieva C, et al. Intensive vs clinical follow-up after treatment of primary breast cancer: 10-year update of a randomized trial. National Research Council Project on Breast Cancer follow-up. JAMA 1999;281:1586.

9. Impact of follow-up testing on survival and health-related quality of life in breast cancer patients. A multicenter randomized controlled trial. The GIVIO Investigators. JAMA 1994;271:1587-1592.

10. Rosselli Del Turco M, Palli D, Cariddi A, et al. Intensive diagnostic follow-up after treatment of primary breast cancer. A randomized trial. National Research Council Project on Breast Cancer follow-up. JAMA 1994;271:1593-1597.

11. Rosselli Del Turco M, Palli D, Cariddi A. It is now the age to define the appropriate follow-up of primary breast cancer patients. J Clin Oncol 1994;12:1996-1997.

12. Rojas MP, Telaro E, Russo A, et al. Follow-up strategies for women treated for early breast cancer. Cochrane Database Syst Rev 2000:CD001768.

13. Moschetti I, Cinquini M, Lambertini $M$, et al. Follow-up strategies for women treated for early breast cancer (review). Cochrane Database Syst Rev 2016:CD001768.

14. State Cancer Profiles. Screening and Risk Factors Table. National Cancer Institute Web site. Available at: https://statecancerprofiles.cancer.gov/risk/ index.php?topic $=$ women $\&$ risk $=v 06 \&$ race $=00 \&$ datatype $=0 \&$ type $=$ risk $\& s$ ort VariableName $=$ default $\&$ sortOrder $=$ default - results. Accessed June 10, 2018.

15. Use of mammography among women aged 40 and over, by selected characteristics: United States, selected years 1987-2013. Available at: https://www.cdc.gov/nchs/data/hus/2015/070.pdf. Accessed June 10, 2018.

16. Larsen K, Merlo J. Appropriate assessment of neighborhood effects on individual health: integrating random and fixed effects in multilevel logistic regression. Am J Epidemiol 2005;161:81-88.

17. Merlo J, Chaix B, Ohlsson $\mathrm{H}$, et al. A brief conceptual tutorial of multilevel analysis in social epidemiology: using measures of clustering in multilevel

logistic regression to investigate contextual phenomena. J Epidemiol Community Health 2006;60:290-297.

18. Hosmer DW, Lemeshow S. Applied Logistic Regression. New York, NY: Wiley; 2000.

19. Centers for Disease Control and Prevention. BRFSS 2013 Survey Data and Documentation. Available at: https://www.cdc.gov/brfss/annual_data/ annual_2013.html. Updated June 23, 2014. Accessed June 22, 2018.

20. Sullivan R, Peppercorn J, Sikora K, et al. Delivering affordable cancer care in high-income countries. Lancet Oncol 2011;12:933-980.

21. Bergstrom R. Drivers of the cost of cancer care. Lancet Oncol 2012;13:1415.

22. Yang Y, Czernin J. Contribution of imaging to cancer care costs. J Nucl Med 2011;52(Suppl 2):86S-92S.

23. Pan H, Gray R, Braybrooke J, et al. 20-Year risks of breast-cancer recurrence after stopping endocrine therapy at 5 years. $\mathrm{N}$ Engl J Med 2017;377:1836-1846.

24. Panageas KS, Sima CS, Liberman L, Schrag D. Use of high technology imaging for surveillance of early stage breast cancer. Breast Cancer Res Treat 2012;131:663-670.

25. Salloum RG, Hornbrook MC, Fishman PA, et al. Adherence to surveillance care guidelines after breast and colorectal cancer treatment with curative intent. Cancer 2012;118:5644-5651.

26. Hahn EE, Tang T, Lee JS, et al. Use of posttreatment imaging and biomarkers in survivors of early-stage breast cancer: inappropriate surveillance or necessary care? Cancer 2016;122:908-916.

27. Paluch-Shimon S, Pagani O, Partridge AH, et al. Second international consensus guidelines for breast cancer in young women (BCY2). Breast 2016;26:87-99.

28. Mold JW, Stein HF. The cascade effect in the clinical care of patients. N Engl J Med 1986;314:512-514.

29. Brodersen J, Siersma VD. Long-term psychosocial consequences of falsepositive screening mammography. Ann Fam Med 2013;11:106-115.

30. Brenner DJ, Hall EJ. Computed tomography-an increasing source of radiation exposure. N Engl J Med 2007;357:2277-2284.

31. Brenner DJ, Hall EJ. Risk of cancer from diagnostic X-rays. Lancet 2004;363:2192; author reply 2192-2193.

32. Brenner DJ, Elliston CD. Estimated radiation risks potentially associated with full-body CT screening. Radiology 2004;232:735-738.

33. Brenner DJ. Radiation risks potentially associated with low-dose CT screening of adult smokers for lung cancer. Radiology 2004;231:440-445.

34. Nuclear Research Council of the National Academies. Health Risks From Exposure to Low Levels of Ionizing Radiation. BEIR VII Phase 2. Washington, DC: National Academy of Sciences; 2006.

35. Kneale GW, Mancuso TF, Stewart AM. Hanford radiation study III: a cohort study of the cancer risks from radiation to workers at Hanford (1944-77 deaths) by the method of regression models in life-tables. Br J Ind Med 1981;38:156-166.

36. Brouwers MC, Vukmirovic M, Tomasone JR, et al. Documenting coordination of cancer care between primary care providers and oncology specialists in Canada. Can Fam Physician 2016;62:e616-625.

37. Jiang L, Lofters A, Moineddin R, et al. Primary care physician use across the breast cancer care continuum: CanIMPACT study using Canadian administrative data. Can Fam Physician 2016;62:e589-598. 
38. van Helmondt SJ, van der Lee ML, de Vries J. Study protocol of the CAREST-trial: a randomised controlled trial on the (cost-) effectiveness of a CBT-based online self-help training for fear of cancer recurrence in women with curatively treated breast cancer. BMC Cancer 2016;16:527.

39. Petrie KJ, Myrtveit SM, Partridge AH, et al. The relationship between the belief in a genetic cause for breast cancer and bilateral mastectomy. Health Psychol 2015;34:473-476.

40. Wapnir IL, Kurian AW, Lichtensztajn DY, et al. Rising bilateral mastectomy rates among neoadjuvant chemotherapy recipients in California from 1998 to 2012. Ann Surg 2017;266:353-360.

41. Abbott A, Rueth N, Pappas-Varco S, et al. Perceptions of contralateral breast cancer: an overestimation of risk. Ann Surg Oncol 2011;18:3129_ 3136.

42. Giuliano AE, Boolbol S, Degnim A, et al. Society of Surgical Oncology: position statement on prophylactic mastectomy. Approved by the Society of Surgical Oncology Executive Council, March 2007. Ann Surg Oncol $2007 ; 14: 2425-2427$.
43. Schwartz MD, Lerman C, Brogan B, et al. Impact of BRCA1/BRCA2 counseling and testing on newly diagnosed breast cancer patients. J Clin Oncol 2004;22:1823-1829.

44. Tuttle $\mathrm{T}$, Habermann E, Abraham A, et al. Contralateral prophylactic mastectomy for patients with unilateral breast cancer. Expert Rev Anticancer Ther 2007;7:1117-1122.

45. Katz SJ, Morrow M. Contralateral prophylactic mastectomy for breast cancer: addressing peace of mind. JAMA 2013;310:793-794.

46. Lo SS, Mumby PB, Norton J, et al. Prospective multicenter study of the impact of the 21-gene recurrence score assay on medical oncologist and patient adjuvant breast cancer treatment selection. J Clin Oncol 2010;28:1671-1676.

47. Siminoff LA, Fetting JH. Effects of outcome framing on treatment decisions in the real world: impact of framing on adjuvant breast cancer decisions. Med Decis Making 1989;9:262-271.

48. Siminoff LA, Fetting JH. Factors affecting treatment decisions for a lifethreatening illness: the case of medical treatment of breast cancer. Soc Sci Med 1991;32:813-818.

See JNCCN.org for supplemental online content.

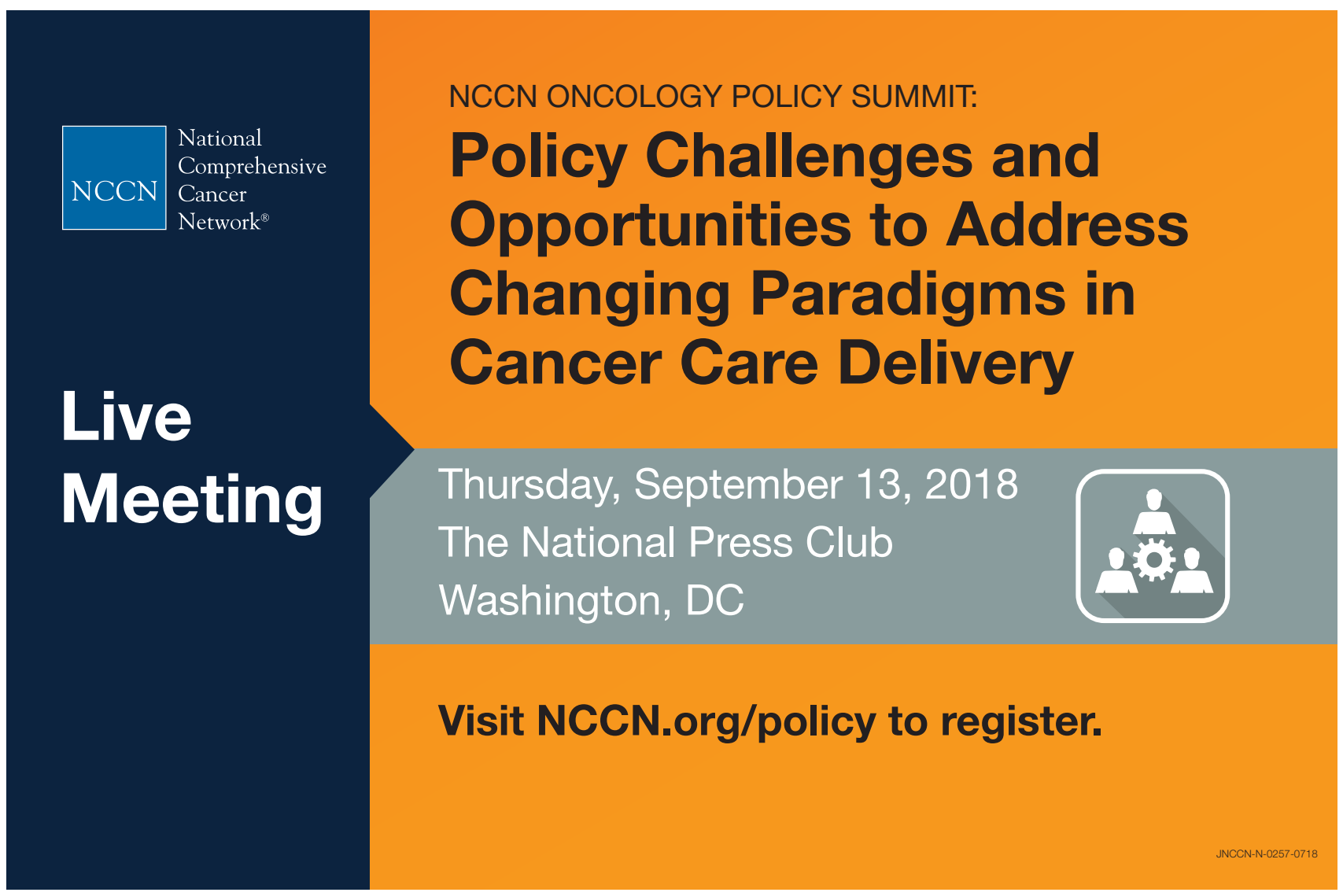

